\title{
Zum Stellenwert von Betroffenheit, Öffentlichkeit und Deliberation im empirical turn der Medizinethik
}

\author{
Silke Schicktanz
}

Online publiziert: 26. August 2009

(C) Die Autor(en) 2009. Dieser Artikel ist auf Springerlink.com mit Open Access verfügbar.

Zusammenfassung Für die Medizinethik liegt ein großes Potential sozialempirischer Forschung in der Erhöhung der Kontextsensitivität, dem Sichtbarmachen von sozialen und institutionellen Rollen und dem Einbringen von Stimmen, die bislang zu wenig gehört worden sind. Diese Möglichkeiten bergen jedoch auch das Risiko, dass Deliberation und Argumentation durch Umfragen und Meinungserhebungen ersetzt werden. Der in den Sozialwissenschaften einsetzende participatory turn gibt Anlass, Anliegen und Methoden klassischer sozialempirischer Vorgehensweisen aus normativer Sicht zu hinterfragen. Eine Auseinandersetzung mit Konzeptionen von Betroffenheit, Öffentlichkeit und Expertise ist nicht nur aus methodologischen, sondern gerade auch aus normativen Gründen wichtig. Für die ethische Relevanz sind dabei die Idee der Argumentation und demokratietheoretische Überlegungen besonders erörterungswürdig. Wenn Bioethik Teil eines auch öffentlich verankerten Diskurses sein soll, ist die Frage nach der angemessenen Form der Berücksichtigung bestimmter Perspektiven ebenso im Kern bioethischer Reflexion zu verorten wie die Rechtfertigung spezieller ethischer Normen.

Schlüsselwörter Empirische Ethik · Partizipation · Öffentlichkeit · Diskursethik · Kollektive Rationalität · Umfragen

The relevance of affected persons, the public, and deliberation for the empirical turn in medical ethics

Abstract Definition of the problem One important potential of socio-empirical research is the increase of context sensitivity, the detection of social and institutional roles and the positioning of so far silent voices. However, there is a risk that socio-empirical surveys

S. Schicktanz $(\bowtie)$

Abteilung Ethik und Geschichte der Medizin, Universitätsmedizin Göttingen

Humboldtallee 36, 37073 Göttingen, Deutschland

E-Mail: silke.schicktanz@medizin.uni-goettingen.de 
and opinion polls replace deliberation and argumentation. Thus, the participatory turn as recently observed in the social sciences gives reason to reflect upon socio-empirical methods. Arguments A critical reflection with concepts of being affected, the public and expertise is important from a methodological as well from a normative point of view. This is especially true for any ethical approach that basically shares the importance of argumentative deliberation and democratic rules in a society. Conclusion If bioethics is seen as part of a public discourse the question of adequate representation of specific perspectives and of different views is as important as the justification of specific norms. Both are at least a core request of bioethical reflection.

Keywords Empirical ethics $\cdot$ Participation $\cdot$ Public $\cdot$ Discourse ethics $\cdot$ Collective rationality $\cdot$ Surveys

\section{Einleitung}

Die aktuelle Diskussion um den empirical turn in der Bioethik entbehrt nicht einer gewissen Ironie: Hatte sich die frühe Bioethik der 1970-80er Jahre nicht sehr darum bemüht, gerade auf Distanz zu den empirischen Wissenschaften zu gehen? Diese methodologische Abgrenzung war zugleich ein wichtiger Schritt der Distanzierung von den Expert(inn)en, deren Handeln ethisch reflektiert, oft problematisiert und nicht selten kritisiert werden sollte (also z.B. von Ärzt(inn)en, Ingenieur(inn)en oder Naturwissenschaftler(inne)n). Inzwischen scheint sich das Blatt gewendet zu haben: Der lauter werdende Ruf nach einer empirischen Ethik konterkariert gerade diese Distanzierung und fordert eher Integration und Hybridisierung [21]. Die Zunahme an empirisch ausgerichteter Forschung beinhaltet ein ganzes Sammelsurium an Ansätzen [3]. Diese Forschungsrichtung speist sich u.a. aus der Medizinsoziologie, der zumeist eher qualitativ ausgerichteten Wissenschaftsforschung (sog. Social Studies of Science) und der quantitativen Forschung zu Einstellungen der Öffentlichkeit gegenüber Technik und Medizin (sog. Public Understanding of Science). Auf den ersten Blick sind alle diese Ansätze methodisch und inhaltlich gleichwertig, denn es gibt kein Argument für eine spezielle Empirie; am besten soll alles kontextsensitiv betrachtet werden.

Während die einen diese Entwicklung begrüßen, stellt sich bei anderen ein (meist vielschichtiges) Unbehagen ein. Der pauschale Einwand, dass solche empirischen Untersuchungen generell irrelevant für die angewandte Ethik seien, ist vielfach zu Recht zurückgewiesen worden [2, 6, 22]. Allerdings bleibt strittig, inwiefern empirische Ergebnisse als Begründung für allgemeine moralische Prinzipien dienen können [22]. Hier bleiben je nach urteilsbildungstheoretischen und meta-ethischen Annahmen Differenzen bestehen, deren Auflösbarkeit fraglich erscheint. Aber angesichts der lang gehegten Differenzen zwischen verschiedenen Ethiktheorien stellt dies auch kein neues Phänomen für die (Bio)Ethik-Debatte dar. Eine andere Quelle des oben genannten Unbehagens speist sich - so meine Vermutung - aus der Sorge, dass hierdurch die kritische Distanz zu den Entscheidungsträgern verloren geht. Diese Sorge ist vielleicht nicht ganz unberechtigt und sollte immerhin Anlass zu kritischer Reflexion möglicher hegemonialer Verhältnisse einer Ethik am Krankenbett bieten. Eine dritte Ursache des Missmuts könnte auch die Infragestellung von Expertise darstellen, und zwar sowohl die von Medizin- als auch zunehmend von Ethikexpert(inn)en. Denn einerseits besteht ein Ziel der vermehrten Hinwendung der Medizinethik zur Empirie in der stärkeren Einbeziehung von Patienten- und Laienperspektiven, andererseits taucht dabei aber auch der/die Sozialwissenschaftler(in) als neue/r Experte/Expertin für bioethische Fragen auf. Mit Blick auf die ganze Spannbreite der Bioethik-relevanten sozialempirischen Forschung 
will ich mich hier deshalb auf ein spezifisches Problem konzentrieren: Wessen Perspektive, wessen Interessen und Ansichten sind eigentlich ethisch relevant und warum? Wo liegen die Möglichkeiten und Beschränkungen verschiedener sozialempirischer Zugänge, diese Perspektiven einzuholen und für ethische Reflexion zugänglich zu machen?

Hierfür will ich im ersten Schritt aufweisen, welche Möglichkeiten und Grenzen in dem jeweiligen Zuschnitt der sozialempirischen Forschung für die Medizinethik liegen. Im zweiten Schritt will ich dann aus ethischer Sicht argumentieren, warum die Bioethik - verstanden auch als Teil eines öffentlichen Diskurses - sich gerade für bestimmte deliberative, interaktive und partizipative Formen der empirischen Forschung öffnen sollte. Dabei muss die aus ethischer und empirischer Sicht notwendige Konzipierung von Öffentlichkeit und Betroffenheit zugleich auch problematisiert werden.

\section{Zielsetzungen der Empirie in der Medizinethik}

Ein zentrales, obgleich sehr allgemeines Argument für mehr Empirie ist Kontextsensitivität $[22,18]$. Hierunter kann man ganz allgemein die Annahme zusammenfassen, dass zum (besseren) Verständnis von Aussagen, Normen und Verhaltensweisen konkrete Praxisbedingungen bekannt sein müssen (z.B. der historische, kulturelle oder soziale Hintergrund der Handelnden). Dabei ist zu bedenken, dass alle Ansätze in der angewandten Ethik ein gewisses Maß an Kontextsensitivität erfüllen müssen - nicht nur solche, die sich das programmatisch auf die Fahne geschrieben haben [22]. Einige Autoren gehen daher so weit, alle Bereichsethiken per se als ethisch-politische Diskurse aufzufassen [15]. So bestehen eigentlich immer Wechselwirkungen zwischen moralisch-ethischen und praktisch-politischen Debatten, denn zum einen sind die Gegenstände bioethischer Debatten häufig public issues und zum anderen bedeutet die notwendige Pragmatisierung und Konkretisierung ethischer Überlegung, auf spezielle Bedingungen Bezug zu nehmen (vgl. den Beitrag von Julia Dietrich in diesem Heft). Alle Theorien angewandter Ethik bauen - manchmal mehr, manchmal weniger explizit - auf grundlegenden sozialtheoretischen und anthropologischen Annahmen auf [8], die die Akteure z.B. entweder als sozial gebunden oder ungebunden oder als jeweils kontextspezifisch vernetzt konzipieren [34]. ${ }^{1}$ Die ethische Relevanz des Kontexts kann zum einen in der Konkretisierung von Problemwahrnehmung und Handlungsumsetzung (Spezifikation von konkreten Situationen, wie z.B.: „Im Fall der potentiellen postmortalen Organentnahmen bei Kindern sind immer die Eltern einzubeziehen, weil...") bestehen. Zum anderen kann sie auch in der Kontextualisierung moralischer Begründung bestehen (Abwägung und Relativierung zu anderen Normen, wie z.B.: „Die freiwillige Entscheidung für eine Lebendorganspende hängt von der Information und Aufklärung über Chancen und Risiken ab."). Letzteres weist nur dann eine gewisse Nähe zum ethischen Relativismus auf, wenn ethische Positionen ausschließlich als abhängig vom kulturellen Kontext gedeutet, formuliert und begründet werden (,Das in Asien existierende Vorrecht der Familie erlaubt dort zu Recht die Einschränkung der persönlichen Freiwilligkeit bei einer Organspende.“").

Ich will am Beispiel der ethischen Diskussion zur Lösung des Organmangels ausführen, wie diese Art der Kontextualisierung aussehen kann. Dabei will ich mich exemplarisch auf

\footnotetext{
${ }^{1}$ Diese soziale und institutionelle Einbettung der Akteure muss dann aus ethischer Sicht einfließen, wenn Konzepte wie Selbstbestimmung und Abgrenzung sowie Auswirkungen auf Rechte Dritter und Verantwortung gegenüber indirekt Betroffenen (und damit die Verhältnisse und Bindungen) angemessen ausdifferenziert werden sollen.
} 
die prominente Studie von Breyer et al. [4] beziehen, die u.a. als einzig ethisch-rechtlich relevante Literatur in der Stellungnahme des Nationalen Ethikrats: „Die Zahl der Organspenden erhöhen“ von 2007 zitiert wird. Die Autoren nehmen an, dass sich jeder Vorschlag, wie der Organmangel zu beheben sei, am öffentlichen Problembewusstsein orientiere müsse, weil andernfalls alle Strategien scheitern würden. Sie bauen damit also vor allem auf die Annahme, dass der Kontext besonders entscheidend für die Handlungsumsetzung sei. ${ }^{2}$ Dabei suggerieren die Autoren mit ihrer Kritik an kleinen Fallzahlen und niedrigen Antwortraten ([4], S. 140 unten), dass nur Repräsentativität das ausschlaggebende Kriterium für die Validität von sozialempirischen Untersuchungen für diese Form der Kontextualisierung sei. Implizit wird hiermit die These vertreten, dass im Rahmen eines ethischen Überlegungsgleichgewichts nur solche ethisch entwickelten Handlungsstrategien sinnvoll sind, die mit der Mehrheitsmeinung der Öffentlichkeit übereinstimmen. Entsprechend versuchen die Autoren anhand einer ganzen Reihe von ausschließlich quantitativen Umfragen ([4], S. 143ff.) ihre These zu untermauern, dass die Organspende und -transplantation sich einer überwältigenden öffentlichen Akzeptanz erfreue ([4], S. 141 unten). Zur weitläufigen Akzeptanz des Hirntods wurden allerdings vor allem Umfragen bei Ärzten herangezogen. Hingegen wurde eine Umfrage, die in der allgemeinen Öffentlichkeit eine sehr geringe Häufigkeit für die Gleichsetzung des Hirntods mit dem Tod des Menschen herausfand, als „schwierig zu interpretieren“ gedeutet ([4], S. 146). Die Autoren interpretieren weiterhin die widersprüchlichen Befunde einer generell häufig genannten grundsätzlichen Befürwortung der Organtransplantation wie auch einer prinzipiellen Spendebereitschaft bei $2 / 3$ der Bevölkerung und das zugleich seltene Besitzen von Organspendeausweisen als Hinweise für die reine „Bequemlichkeit“ oder „Gedankenlosigkeit“ in der Öffentlichkeit. Entsprechend schlussfolgern sie, dass eine Einführung der Widerspruchslösung gerechtfertigt wäre, obwohl sie selbst Umfragen zitieren, die explizit nach verschiedenen Verfahren der Zustimmung (also Zustimmungslösung vs. Widerspruchslösung) fragten und eine ausschließlich hohe Resonanz für die enge und etwas weniger für die weite Zustimmungslösung fanden. Problematisch erscheint besonders, dass die Ja/Nein-Antworten zu verschiedenen quantitativen Umfragen als „normative Positionen [...] im Alltagsbewusstsein“ bezeichnet werden ([4], S. 177). Zum einen ist auffällig, dass in den genannten Umfragen nie nach der Perspektive der Patienten gefragt wurde: Würden sie z.B. Organe aus einem Organhandel überhaupt akzeptieren? Sind sie zu 100\% bereit, ihre Organe für andere zu spenden? Zum anderen fällt auf, dass Ergebnisse aus qualitativen Interviewstudien wie beispielsweise von Hauser-Schäublin et al. [14] nicht berücksichtigt wurden. Sie hätten die große Ambivalenz und Zurückhaltung im Umgang mit Organspenden im konkreten Fall, z.B. einer Stellvertreterentscheidung durch die Angehörigen, wesentlich besser erklärt als lediglich „Bequemlichkeit" und „Gedankenlosigkeit“.

Nicht trivial erscheint mir daher die Forderung nach einer spezifischen ethischen Rechtfertigung, wen man beforscht bzw. wessen Perspektive besonders wichtig ist und ggf. unter Bedingungen knapper Forschungsressourcen gar vorrangig zu beforschen wäre. Daher halte ich es auch für normativ problematisch, wenn zum Ziele der Kontextualisierung vorrangig die Perspektive der Entscheider (d.h. der Ärzte und des Pflegepersonals) erhoben wird, und nicht die der Patienten und Angehörigen (vgl. auch den Beitrag von Musschenga). Weiterhin ist es meines Ermessens nicht beliebig, ob man quantitative deskriptive oder qualitative argumentative Methoden im Rahmen dieser empirischen Forschung einsetzt.

\footnotetext{
${ }^{2}$ Bedauerlicherweise führen die Autoren nicht genauer aus, ob sie ihr Argument ausschließlich als praktisches Klugheitsargument verstehen, oder - was aufgrund ihrer Gesamtargumentation etwas näher liegt - sie es eher normativ deuten, dass jede praktisch-ethische Lösung auch umsetzbar sein sollte.
} 
Die Erhöhung von Kontextsensitivität mittels empirischer Untermauerung kann insbesondere dort wichtig sein, wo Grundannahmen zu kontextuell variierenden Unterschieden in der institutionellen Einbindung und sozialen Rollenübernahme der unterschiedlichen Akteure vorliegen. Gerade innerhalb der Medizinethik dominierte ja lange Zeit die Vorstellung (und sie ist auch nicht gänzlich obsolet), dass im Zentrum ethischer Überlegungen allein das Individuum bzw. die Interaktion zwischen zwei Individuen zu stehen habe, nämlich zwischen Arzt/Ärztin und Patient/in. Diese Konzeptualisierung des Arzt-Patienten-Verhältnisses als die zentrale menschliche Interaktion und damit der Fokus medizinethischer Reflexion hat sich allerdings als unzureichend erwiesen. So sind bei Entscheidungen in der Reproduktionsmedizin oder zum Schwangerschaftsabbruch, am Lebensende, zur Organspende und zum Umgang mit genetischem Wissen nicht nur Individuen, sondern auch ganze Kollektive (z.B. Familien, Patientengruppen, Professionsorganisationen, Behindertengruppen, Beitragszahler, die Öffentlichkeit etc.) als solche involviert, ohne dass es sich dabei um definierte, rein akkumulierte Einzelinteressen handelt. Blendet die ethische Reflexion diese Dimension der sozialen Praxis systematisch aus, so ist dies nicht nur theoretisch unzureichend, sondern auch ihr Anspruch, praktisch zu werden, d.h. Einfluss auf die zukünftige Praxis zu haben, im Kern fragwürdig. Die Erweiterung individualethischer Perspektiven um die kollektive Dimension ist dabei für die Ethiktheorie an sich nichts Neues, denkt man an die sozial- und politisch-ethischen Gerechtigkeitsdebatten zur nationalen und internationalen Verteilung von Gesundheitsgütern. Alle Auseinandersetzungen über Fragen des kollektiven Lebens müssen sich heutzutage entsprechend auch demokratietheoretischen Überlegungen stellen - und sei es nur, um eine expertokratische, deliberative oder rein repräsentative Form der Auseinandersetzung zu verteidigen [25].

\section{Der participatory turn: Zur Rolle von Öffentlichkeit für die Bioethik}

Innerhalb der empirischen Sozialwissenschaften finden wichtige Debatten zur Methodologie und zum Theorie-Praxis-Verhältnis statt, die sich mit dem participatory turn beschäftigen. Hierunter wird die Hinwendung zur (aktiven) Beteiligung der Öffentlichkeit in den Sozialwissenschaften verstanden: Die Sozialwissenschaften erforschen nicht nur (akademisch) die soziale Ordnung in Gesellschaften, sondern nehmen selbst Einfluss auf diese [11] und sollen nun - umgekehrt - die Öffentlichkeit auch direkter als bisher in die eigene Forschung einbinden. Dabei kann man sich als ironischer Kommentator, Übersetzer, Reformer oder gar Revolutionär in Bezug auf gesellschaftlichen Umgang mit Wissen verstehen [13]: Entsprechend reicht das Methodenspektrum von quantitativen Befragungen von Patienten und Laien über Interaktionsforschung mit Betroffenen bis hin zu Bürgerforen oder sogenannten Citizen Juries. Immer geht es um die direkte oder indirekte Einbindung der Meinung oder Erfahrungen der Nicht-Experten. Ähnlich wie die Sozialwissenschaften (insbesondere die Public-Understanding of Science-Forschung) ist auch die Bioethik kein rein akademisches Vergnügen, sondern Teil des öffentlichen Diskurses [7, 15]. Dies befördert die - aus akademischer Bioethik-Sicht freilich oft unerfreuliche, weil unzutreffende - Gleichsetzung von Bioethik mit politischen Kommissionen oder so genanten Bioethik-Richtlinien [19, 16].

Der participatory turn wird durchaus als eine selbstkritische Wendung der Sozialwissenschaften diskutiert, in der die eigenen Experten-Rollen und die sozialpolitische Funktion von z.B. Life Science-Forschung problematisiert werden [1]. Die theoretischen Annahmen darüber, was eine Öffentlichkeit überhaupt ist, wie sie jeweils konstruiert wird und was die jeweiligen Methoden leisten können, werden kontrovers diskutiert [10]. Die Rolle von Partizipation spielt dabei in der Forschungsagenda einiger Länder (z.B. Großbritannien) eine so 
gravierende Rolle, dass inzwischen einige Autoren von der „Tyrannei der Partizipation“ sprechen (allerdings mit Bezug auf Stadtplanung und Lokalpolitik) [5]. Aus ethischer Sicht ist die zunehmende Hinwendung zur Partizipation in den sozialempirischen Wissenschaften besonders interessant, weil man hierin eine normative Wende in den empirischen Wissenschaften erkennen kann. Denn schließlich liegen hier normative, meist implizit demokratietheoretische Rechtfertigungen zu Grunde, warum und wie die Öffentlichkeit zu beforschen ist.

Die Schnittstelle von empirischer, partizipativer und medizinethischer Forschung ${ }^{3}$ ist daher von besonderer Bedeutung. An sie lassen sich grundlegende Überlegungen zum Verhältnis von Bioethik und Demokratie anschließen. Im Kern besagt meine folgende Argumentation, dass für die Idee der wechselseitigen Anerkennung von Rechten oder Interessen, wie sie meinem Verständnis nach in allen liberalen, auf Selbstbestimmung ausgerichteten Ansätzen gefordert wird, die kommunikative Verständigung mittels Argumenten eine tragende Rolle spielt. Dies betrifft eigentlich alle kognitivistisch ausgerichteten Ethikansätze, ${ }^{4}$ die für die Klärung kollektiver Angelegenheiten eine nicht rein-monologische Reflexion favorisieren (wie z.B. explizit die Diskursethik oder z.T. auch der Präferenzutilitarismus Peter Singers [32], aber ebenso andere prozedurale oder kommunitaristische Ansätze). Für sie kann eine öffentliche, demokratische Auseinandersetzung zu bioethischen Fragen nicht nur ein Add-on, ein zierendes Sahnehäubchen moderner Gesellschaften sein, sondern muss im Kern gefordert und gefördert werden. Empirie sollte aus dieser Sicht für die Bioethik gerade dort ins Spiel kommen, wo die monologische Reflexion durch Perspektivenpluralismus aufgebrochen und kritisch geprüft werden muss. Und zugleich geht es um die Verankerung von Bioethik als eines Elementes moderner, demokratisch ausgerichteter Politik.

Ich setze dabei voraus, dass die meisten bioethischen Fragen immer schon eine kollektive Dimension beinhalten und nicht eine rein individuelle Angelegenheit sind. Bioethische Problemstellungen zeichnen sich häufig gerade dadurch aus, dass die Einordnung als rein individualistische Frage der eigenen Lebensführung oder als soziales Problem mit Verpflichtungen gegenüber Dritten extrem strittig ist. ${ }^{5}$

\section{Grenzen der monologischen Reflexion}

Ethische Reflexion und Urteilsbildung knüpfen an grundlegende Prämissen der Argumentationstheorie an. Dabei bleibt in vielen Ansätzen unklar, woher die Argumente kommen müssen, ob also eine interne Generierung derartiger Argumente - mittels der eigenen, individuellen Vernunft - ausreicht. Ein stark vernunftorientierter Ansatz der Ethik in der Kant'schen Tradition würde es der Vernunft des einzelnen Menschen im Prinzip zutrauen (müssen), sich die verschiedenen Pro- und Contra-Argumente selbst vor Augen zu führen. Dabei können immer noch Klugheitsgründe dafür sprechen, Stellungnahmen Dritter (zum Beispiel des akademischen oder öffentlichen Diskurses) als Quelle und Inspiration für weitere, neue Argumente zu nutzen. Vertritt man jedoch aus epistemologischer Sicht, dass die individuelle Vernunft stark kognitiven, örtlichen und zeitlichen Begrenzungen unterliegt, so folgt daraus, diesen individuellen Zugang prinzipiell in Frage zu stellen. Dabei kann man sowohl kognitive als auch soziale Determinanten als mögliche Gründe anführen. Aus

\footnotetext{
${ }^{3}$ Wobei empirisch-partizipative Methoden sich durch explizite diskursive und argumentative Elemente von anderen empirischen qualitativen Methoden unterscheiden. Hierunter wären z.B. Fokusgruppendiskussionen, geleitete und dokumentierte Internetforen, Dialog-Verfahren, Szenario-Workshops etc. zu rechnen.

${ }^{4}$ Siehe [26] zur Unterscheidung von kognitivistischen und non-kognitivistischen Ansätzen.

5 Ich habe versucht, dies an anderer Stelle zu zeigen [28].
} 
ethisch-normativer Sicht wäre es gerade eine Verantwortungsperspektive, die im strikten Sinne die Einholung anderer Meinungen und Sichtweisen einfordert.

Hierzu ist es notwendig, kurz an die Annahme einer moralischen Ursituation zu erinnern, die eigentlich jeder Ethikargumentation zugrunde liegt. Hiermit meine ich die Idee einer Begründung für die moralische Rücksicht auf Andere. Sie kann z.B. entweder aufgrund von wechselseitigem, individuellem Vorteil im Naturzustand (nach Locke) erfolgen, aus vernünftiger Einsicht in die Universalgütigkeit des Nicht-Instrumentalisierungsgebots (nach Kant) oder aus dem Wunsch nach sozialer Kooperation und gleichzeitiger Unwissenheit bzgl. des möglichen eigenen sozialen Status (nach Rawls) [23, 35]. Alle rationalistischen Konzeptionen einer ethischen Argumentation gehen mehr oder minder davon aus, dass wir in der ethischen Diskussion von eigenen Interessen abstrahieren und die Sicht des Anderen mit in den Blick nehmen müssen. Ein gewisses Maß an Unparteilichkeit muss angenommen werden, die sich in Form eines argumentativen Rollenwechsels darstellt; außerdem sollten wir für eine einvernehmliche Lösung argumentieren, statt Gewalt oder Macht anzuwenden. Wie Martha Nussbaum herausstellt, bauen jedoch alle diese Ansätze im Kern auf der anthropologischen (bzw. naturalistischen) Annahme auf, dass alle Menschen frei, gleich und autark sind [23]. Doch genau des rationalen Universalisierungsmoments ethisch-kritischer Prüfung bedarf es, dass wir diese Annahmen zum gleich, frei und autark sein hinterfragen bzw. kontextualisieren, also in welchen Abhängigkeiten ein Mensch sich befinden kann: Ist es wirklich so, dass wir uns (z.B. als jemand Gesundes, kognitiv weitgehend Funktionierendes, mit einem westlichen, sozial abgesicherten Hintergrund) angemessen und ausreichend in die Situation von jemandem hineinversetzen können, der einen anderen kulturellen Hintergrund hat, in materieller und sozialer Unsicherheit lebt, ein anderes Geschlecht hat, chronisch krank oder behindert ist? Aus einer ethisch-rationalistischen Perspektive ist klar, dass wir es versuchen sollten - dem epistemiologischen Skeptizismus zufolge ist es sehr fragwürdig, ob wir es ausreichend leisten können.

Ich glaube, dass hier nun die Diskursethik einen enorm wichtigen Beitrag zu der modernen Diskussion der Probleme ethischer Urteilsfindung leistet. ${ }^{6}$ Zwar teilt die Diskursethik die Idee der Argumentation und der Kommunikation als notwendige Voraussetzungen für ethische Urteilsfindung mit vielen anderen modernen Ethikansätzen. Sie hebt jedoch den Gedanken hervor, dass die Prüfung der Gültigkeit der Argumente prinzipiell nicht einer einzelnen Person überlassen werden kann, sondern gemeinschaftlich stattzufinden hat [24]. Somit ergeben sich normative Regelungen für den praktischen Diskurs, in dem wiederum prozedural vorgeschlagene Normen geprüft werden sollen. Dabei untermauern zugleich soziologisch-politische Beobachtungen (Kommunikation unterliegt bestimmten sozialen Machtverhältnissen und Asymmetrien) und daraus resultierende normative Argumente (solche Machtverhältnisse müssen ausgehebelt werden, um freies Argumentieren zu erlauben) die Idee, dass es eine kollektive Verständigung zur Lösung ethischer Konflikte braucht [33]. Die Diskursethik vertritt dabei ein partizipatorisches Ideal, welches der Exklusion von Betroffenen aus einem ethischen Diskurs skeptisch gegenüber steht [36]. Konsequenterweise wäre es notwendig, die Inklusion der Betroffenen zu fordern. Die Umsetzung in reale Diskurse ist eine Möglichkeit (die zwar aus Sicht der meisten Diskursethiker keine notwendige Bedingung darstellt, aber in der Tendenz mehr als nur wünschenswert ist), um den

\footnotetext{
${ }^{6}$ Dies wird in seiner Geltung für die Medizinethik m.E. unterschätzt. In anderen Anwendungsbereichen wie der Technikethik wird hingegen wesentlich länger die normative Rolle und das Potenzial von Öffentlichkeit und kollektiver Deliberation diskutiert [33]. Ein Grund für diese Ausblendung mag, wie oben angedeutet, in der unplausiblen Fokussierung der medizinethischen Kernsituation auf das interindividuelle Arzt-PatientenVerhältnis liegen. Auch andere, kommunitaristische Ansätze oder Autoren wie Charles Taylor argumentieren in ähnlicher Weise [34].
} 
unterschiedlichen Interessen und Wertorientierungen der Betroffenen Rechnung zu tragen. Dabei vertrauen die meisten diskursethisch orientieren Autoren, wie andere rationalistische Ethiken auch, letztlich auf die Vernünftigkeit der Diskursteilnehmer, die unterschiedlichen Argumente zu prüfen und erst nach dieser Prüfung einen Konsens zu erzielen. Die Legitimation erfolgt durch Rationalität, welche wiederum durch Argumentation gesichert wird. Diese Rationalität muss jedoch intersubjektiv hergestellt werden und kann nicht ausschließlich intrasubjektiv überprüft werden. Der prozedurale Charakter der Diskursethik kann leicht so interpretiert werden, dass diese Ethikkonzeption einen überhöhten, kaum einlösbaren Anspruch an die soziale Realität stellt und derartige praktische (ideale) Diskurse nicht erfüllt werden können. ${ }^{7}$ Man könnte daher davon ausgehen, dass ein (wissenschaftlicher) Diskurs zwischen Ethikexperten den Diskurs zwischen Betroffenen ersetzen kann (oder er sei sogar besser, weil er weniger anfällig für Manipulation sei). Auch wenn ich an dieser Stelle diesen Streit nicht als zu Gunsten der Expertise entschieden ansehe, ${ }^{8}$ glaube ich, dass hier genau die Diskussion um die empirische Forschung in der Bioethik in den Vordergrund tritt: Die empirische Untermauerung der deskriptiv-ethischen Annahmen innerhalb einer speziellen Kontroverse erscheint mir ganz wesentlich für die Plausibilitätserzeugung, nämlich um Aussagen über die Öffentlichkeit oder die Betroffenen zu plausibilisieren.

Nehmen wir das oben erwähnte Beispiel: Für die Rechtfertigung der Einführung einer Widerspruchslösung bei der Organspende wird das Argument angeführt, dass von einer grundsätzlichen mehrheitlichen Einwilligung der Bevölkerung und der potentiellen Empfänger derart gewonnener Organe auszugehen ist [4]. Dies ist ein zentrales Zusatzargument der Autoren, um die Widerspruchslösung mit der liberalen, grundgesetzlich abgesicherten Position vereinbaren zu können, dass niemand gegen seinen Willen zum postmortalen Organspender werden soll (sonst würde man die so genannte Notstandslösung favorisieren). Allerdings ist hier ein wichtiger kritischer Punkt anzumahnen: Die abgefragte Einzelmeinung der Befragten wird hier in aufsummierter Form als kollektiver Wille der Öffentlichkeit dargestellt. Dabei handelt es sich also im strengen Sinne um die demokratietheoretische Annahme, dass die Normen bzw. Lösungsansätze (rechtlich-ethische) Gültigkeit erlangen können/sollen, die von der Mehrheit der Bürger favorisiert werden. Allerdings ist die Majoritätsregel nicht per se der eigentliche Kern demokratietheoretischer Überlegungen, sondern es gehören hierzu genauso Rücksichtsnahmen auf Minderheiten. Außerdem ist die aufaddierte Mehrheitsmeinung nicht mit einem auf Argumentationsaustausch resultierenden Konsens gleichzusetzen.

Sozialempirische Forschungen zur Erhärtung von ethischen Prämissen zur gesellschaftlichen Umsetzung sind insofern nur ein Schritt in der Gesamtargumentation. Sie beruhen alle auf spezifischen normativ aufgeladenen Deutungen von Öffentlichkeit und Gesellschaft. Im besten Falle sind diese Überlegungen demokratisch orientiert und gehen also davon aus, dass auch bioethische Normen sich den Regeln der Demokratie anzupassen haben. Dies setzt allerdings voraus, dass ein politisch-ethischer Standpunkt zur Demokratietheorie entwickelt werden muss. ${ }^{9}$ Aus Sicht einer rationalistischen, liberalen und zugleich auf Argumentation setzenden Perspektive haben m. E. daher deliberative Theorien ein besonders großes Potential, plausible und kohärente Argumentationen zu sichern. Deliberation meint

\footnotetext{
${ }_{7}$ Diesen verbreiteten Einwand halte ich jedoch für sehr schwach, weil er eigentlich alle rationalistischen Ethiken, die ein ethisches Ideal als allgemeine Orientierung vertreten, treffen würde und somit nur von nichtrationalistischen Perspektiven aus geführt werden kann. Zudem trifft er auch alle Formen der Demokratie, da diese immer als politisches Ideal und nie als empirisches Faktum gesehen werden.

${ }^{8}$ Meine Einwände gegen eine ausschließliche Begrenzung auf den Expertendiskurs siehe [27].

${ }^{9}$ So kann man zwischen elitären, ökonomischen, liberal-pluralistischen, republikanischen, partizipativen oder deliberativen Konzepten unterscheiden [20].
} 
hierbei ganz allgemein eine auf Überlegungen und Argumentation basierte Entscheidungsfindung. Im Gegensatz zur Darstellung von Öffentlichkeit mittels aufsummierter, respräsentativer Meinungsumfragen würde aus der Sicht einer deliberativ-diskursethischen Position interessieren, ob die erwogenen Normen im Rahmen einer diskursiven, argumentativen Erörterung von den Betroffenen geteilt und befürwortet werden. Nur diese Position setzt voraus, dass eine Meinung erst dann Relevanz hat, wenn man davon ausgehen kann, dass sie auf der Erwägung der verschiedenen Perspektiven beruht. Dies setzt nun in gewissem Maße eine aktive Beteiligung am Diskurs, am Argumentationsprozess voraus. ${ }^{10}$ Die Öffentlichkeit bzw. die Betroffenen sind hierbei nicht nur für die politisch-moralisch-pragmatische Umsetzung zu befragen; es geht auch darum, ob die vorgefundenen Argumente von den (z.T. unterschiedlich) Betroffenen rational geteilt werden. ${ }^{11}$ Die normativ begründeten Regeln, wie dieser Diskurs gestaltet werden muss bzw. welche Konditionen - z.B. gerechte und faire Kommunikationsstrukturen - vorab zu schaffen sind, sind - übertragen auf die empirische methodologische Diskussion - nicht gänzlich irrelevant oder trivial. Sie führen nochmals vor Augen, dass die Vorentscheidung, wer als Betroffener gilt und wer nicht, einer expliziten Rechtfertigung bedarf und ein Bias zu vermeiden ist. Gerade das Beispiel der Organtransplantation zeigt, dass im Diskurs oft nur die Patienten, die ein Organ benötigen, als Betroffene konzipiert werden. Hingegen werden potentielle Spender auf der anderen Seite meist als Nicht-Betroffene konzipiert, was jedoch problematisch ist [31].

Berücksichtigt man statt ausschließlich quantitativer Studien auch sozialempirisch-qualitative Forschungen, so wird schon wesentlich plausibler, warum einige der Umfrageergebnisse schwierig zu interpretieren sind. Denn auch Patienten und Laien zeigen in komplexen bioethischen Fragen Inkohärenzen, Ambivalenzen und moralische Unsicherheit [30, 31] - was jedoch am Anfang eines jeden neuen bioethischen Expertendiskurses ebenfalls zu beobachten ist. Hinzu kommt eine Form des Perspektivenpluralismus, die nicht allein auf Meinungsdifferenzen, sondern auf unterschiedliche Formen des Rollenverständnisses, der Erfahrung mit dem medizinischen System oder moralisch-religiöse Grundhaltungen zurückzuführen ist. Allenfalls am Ende von ausgeprägten Ethikdebatten macht es wirklich Sinn, von kanonisierten, konträren Positionen zu sprechen (wie z.B. in der neueren Diskussion um den moralischen Status des Embryos) und somit den Konsens über den Dissens als gerechtfertigten Ausgang solcher Debatten zu würdigen. Diese Bedingung sollte auch zur Bewertung öffentlicher Meinungen angewendet werden.

\section{Demokratische Zumutungen an die Bioethik}

Die Diskussion um empirical ethics scheint mir dort besonders neuartig und relevant zugleich, wo es um die aktive Einbeziehung von Stimmen, Erfahrungen und Positionen

\footnotetext{
${ }^{10}$ Hingegen ist beim Ankreuzen von Ja/Nein-Fragen im Rahmen einer Konsumentenbefragung durch ein Meinungsforschungsinstitut wie Forsa fraglich, ob die Befragten ausführlicher die Pro- und Contra-Argumente der Organspende erwogen haben. Wenn Umfragen also ein Stück weit den öffentlichen Diskurs abbilden sollen, so wäre zumindest sicherzustellen, dass ein solcher Diskurs stattgefunden hat. Dies ist aber in vielen Fällen fraglich. So genügt es z.B. nicht, auf eine ausreichende Medienberichterstattung zu verweisen. Gerade die Gleichsetzung von medialem Diskurs mit öffentlichem Diskurs gilt insbesondere im Bereich komplexer bioethischer Fragen und unter den strukturellen Machtverhältnissen der Wissenschaft als fraglich, weil dort die Medien eher zur Repräsentation hegemonialer Verhältnisse neigen oder ihrer medienökonomischen Logik folgen müssen, statt kritisch verschiedene Positionen zu beleuchten [9, 17].

${ }^{11}$ Dabei gehe ich davon aus, dass auch die noch nicht, nicht mehr oder nie aktiv Teilnehmenden als moralisch relevante Moralobjekte durch Antizipation berücksichtigt werden können (so wie kleine Kinder, geistig Schwerstbehinderte oder auch Tiere, Embryonen etc.).
} 
außerhalb des bisherigen Expertendiskurses geht. Der deliberative Ethikansatz macht zu Recht auf die Frage des Wie und des Wer solcher kollektiv relevanten Entscheidungsfindungen aufmerksam: Wie wurden die Meinungen und Stimmen erhoben, um deskriptive, empirische und normative Prämissen zu kontextualisieren und zu plausibilisieren? Im Sinne einer interdisziplinären Auseinandersetzung kommt man also nicht umhin, wissenschaftstheoretische und methodologische Aspekte von quantitativen und qualitativen empirischen Studien mit zu hinterfragen. Quantitative Studien werden oft noch als der Goldstandard für Erhebungen öffentlicher Meinungen angesehen, weil hier das Gesamtkollektiv im Vordergrund steht. Doch qualitative Forschung kann meist erst leisten, was in quantitativen Umfragen vorausgesetzt wird, nämlich: angemessene Hypothesen zu bilden. Zweitens kann qualitative Forschung mögliche Ambivalenzen aufzeigen und Interpretationsmuster anbieten, da die erhobenen Daten wesentlich komplexere Einsichten in Einstellungen, Zusammenhänge und Argumentationsverläufe geben als quantitative, rein statistisch ausgewertete Umfragen. Drittens bieten zumindest manche qualitative Forschungsansätze die Möglichkeit zum expliziten participatory turn, weil sie aktives Argumentieren und Kommentieren der Teilnehmer(innen) ermöglichen.

Da ethische Forschung - folgt sie den bisher ausgeführten allgemeinen Überlegungen zum Anspruch von rationalistischer Nachvollziehbarkeit - vor allem an Argumenten interessiert ist, sollte sie auch die Argumente der von der Situation durchaus unterschiedlich Betroffenen interessieren. Dass gerade die Betroffenen einen Sonderstatus für die normative Bewertung von moralisch relevanten Aussagen haben, hat mit Dreierlei zu tun [29]: Erstens erfüllt dies am ehesten die in verschiedenen Ethiktheorien immer wiederkehrende Vorstellung, dass die Personen, die von einer Entscheidung konkret betroffen sind bzw. die Konsequenzen tragen, auch selbst mit entscheiden sollen - also eine konkretisierte Form der Selbstbestimmung. Zweitens nährt der epistemologische Skeptizismus Zweifel an der Möglichkeit, alle überhaupt möglichen Aspekte und Argumente im Rahmen einer individuellen, monologisch geführten Vernunfterörterung zu erschließen. Drittens stellt sich die Frage, ob Experten (auch im Kollektiv) in der Lage sind, Alltagsprobleme und -perspektiven ausreichend zu erfassen und zu reflektieren. Zwar muss öffentliche Deliberation nicht ausschließlich auf aktiver Partizipation beruhen, sondern kann ggf. auch auf repräsentative Mechanismen inklusive Experten- und Mediendiskurs vertrauen. Allerdings scheint besonders bei moralischen Konflikten die Frage aufzutauchen, wie Verantwortungsübernahme und Reziprozität hergestellt werden sollen, wenn die Akteure und Adressaten (also die Bürger) nicht aktiv am Entscheidungsfindungsdiskurs beteiligt wurden [12].

Der akademische Ethik-Diskurs muss seine eigenen Grenzen zur sozialen Wirklichkeit anerkennen - das ermöglicht ja unter anderem auch seine Professionalität im Weber'schen Sinne. Zugleich ist es naiv, den akademischen (theoretischen) Diskurs von der Wirklichkeit realer Diskurse als komplett getrennt zu konzipieren. Der ethische Fachdiskurs interagiert bereits mit dem öffentlichen Diskurs (durch Ethikkomissionen, Tagungen und journalistische Arbeiten). Auch empirische Forschung - wenn sie argumentierende, diskursive, partizipative Elemente enthält, sollte also entsprechend als (ein oftmals noch explorativer) Versuch verstanden werden, öffentliche Deliberation zu bioethischen Themen anzuregen. Daher ist die aktive Beteiligung der Betroffenen, sei es durch die reflektierte Bezugnahme auf empirische Studien oder die Förderung eines aktiven, öffentlichen Diskurses, eine Forderung, die m. E. nicht nur eingefleischte Diskursethiker vertreten können, sondern alle, die wirklich an der Steigerung von Rationalität durch Argumentation interessiert sind. Sozialwissenschaftler(inne)n kommt hier die wichtige Aufgabe zu, die Möglichkeiten und Grenzen methodischer Settings zu reflektieren und ggf. neue Methoden zu entwickeln. Der participatory turn bietet interessante Ansätze hierzu. 
Für anregende Diskussionen und wichtige Hinweise danke ich ganz besonders Mark Schweda, Göttingen, und Konrad Ott, Greifswald.

Open Access Dieser Artikel unterliegt den Bedingungen der Creative Commons Attribution Noncommercial License. Dadurch sind die nichtkommerzielle Nutzung, Verteilung und Reproduktion erlaubt, sofern der/die Originalautor/en und die Quelle angegeben sind.

\section{Literatur}

1. Bauer MW, Allum N, Miller S (2007) What can we learn from 25 years of PUS survey research? Liberating and expanding the agenda. Public Understan Sci 16:79-96

2. Birnbacher D (1999) Ethics and social science. Which kind of co-operation? Ethic Theory and Moral Prac 2:319-336

3. Borry P, Schotsmans P, Dierickx K (2005) The birth of the empirical turn in bioethics. Bioethics 19(1):50-71

4. Breyer F, Daele W, Engelhard M, Gubernatis G, Kliemt H (2006) Organmangel. Ist der Tod auf der Warteliste unvermeidbar? Springer, Berlin

5. Cooke B, Kothari U (eds) (2001) Participation: The new tyranny? Zed Books, London

6. Doris J, Stich S (2008) Moral psychology: Empirical approaches. The stanford encyclopedia of philosophy. (http://plato.stanford.edu/entries/moral-psych-emp/ [Access: 8/7/2009])

7. Düwell M (2000) Die Bedeutung ethischer Diskurse in einer wertpluralen Welt. In: Kettner M (Hrsg) Angewandte Ethik als Politikum. Suhrkamp, Frankfurt a.M., S 76-116

8. Düwell M (2005) Sozialwissenschaften, Gesellschaftstheorie und Ethik. Jahrbuch für Wissenschaft und Ethik 10:5-22

9. Gerhards J, Schäfer MS (2006) Die Herstellung einer öffentlichen Hegemonie. Humangenomforschung in der deutschen und der US-amerikanischen Presse. VS, Wiesbaden

10. Gerhardt J, Neidhardt F, Rucht D (1998) Zwischen Palaver und Diskurs. Strukturen öffentlicher Meinungsbildung am Beispiel der deutschen Diskussion zur Abtreibung. Westdeutscher Verlag, Opladen

11. Giddens A (1987) Social theory and modern sociology. Polity Press, Cambridge

12. Gutmann A, Thompson D (1998) Democracy and disagreement. Harvard University Press, Cambridge

13. Hacking I (1999) Was heißt , soziale Konstruktion“? Zur Konjunktur einer Kampfvokabel in den Wissenschaften. Fischer, Frankfurt

14. Hauser-Schäublin B, Kalitzkus V, Petersen I, Schröder I (2001) Der geteilte Leib. Die kulturelle Dimension von Organtransplantation und Reproduktionsmedizin in Deutschland. Campus, Frankfurt a.M.

15. Kaminsky C (2005) Moral für die Politik. Eine konzeptionelle Grundlage der angewandten Ethik. Mentis, Paderborn

16. Kelly SE (2003) Public bioethics and publics: Consensus, boundaries, and participation in biomedical science. Policy, Sci, Technol \& Human Values 28:339-364

17. Kettner M (2006) Medizinethik in den Medien - Befunde und Aufgaben in Theorie und Praxis. Ethik Med 18(4):353-358

18. Krones T (2008) Kontextsensitive Bioethik: Wissenschaftstheorie und Medizin als Praxis. Campus, Frankfurt a.M.

19. Lösch A (2001) Genomprojekt und Moderne. Soziologische Analysen des bioethischen Diskurses. Campus, Frankfurt a.M.

20. Lösch B (2005) Deliberative Politik. Moderne Konzeptionen von Öffentlichkeit, Demokratie und politischer Partizipation. Westfälisches Dampfboot, Münster

21. Molewijk B (2004) Integrated empirical ethics: In search for clarifying indentities. Med Health Car Philos 7:85-88

22. Musschenga AW (2005) Empirical ethics, context sensitivity, and contextualism. J Med Philos 30:467-490

23. Nussbaum M (2006) Frontiers of justice. Disability, nationality, species membership. Harvard University Press, Cambridge MA

24. Ott K (2001) Moralbegründungen - Zur Einführung. Junius, Hamburg

25. Peters B (2001) Deliberative Öffentlichkeit. In: Wingert L, Klaus G (Hrsg) Die Öffentlichkeit der Vernunft und die Vernunft der Öffentlichkeit. Suhrkamp, Frankfurt a.M., S 655-677

26. Ricken F (1998) Allgemeine Ethik. Kohlhammer, Stuttgart

27. Schicktanz S (2008) Politikberatung im Kontext der Medizin. In: Bröchler S, Schützeichel R (Hrsg) Politikberatung. Lucius \& Lucius, Stuttgart, S 47-69 
28. Schicktanz S (2009) Interpreting advance directives: Ethical considerations of the interplay between personal and cultural identity. Health Care Anal 17(2):158-171

29. Schweda M, Schicktanz S (2008) Betroffenheit - Ein Fall von Meinungsvielfalt oder eine Frage des Blickwinkels? In: Michl S, Potthast T, Wiesing U (Hrsg) Pluralität in der Medizin. Alber, Freiburg i.Br., S 235-252

30. Schweda M, Schicktanz S (2008) Public moralities concerning donation and disposition of organs - results of a European cross-cultural study. Camb Q Healthc Ethics 17:308-317

31. Schweda M, Schicktanz S (2009) The spare parts person? Conceptions of the human body and their implications for public attitudes towards organ donation and organ sale. Philos Ethics Humanit Med 4(4): $1-28$

32. Singer P (1994) Praktische Ethik. Reclam, Stuttgart

33. Skorupinski B, Ott K (2000) Technikfolgenabschätzung und Ethik. Vdf, Zürich

34. Taylor C (1997) Cross-purposes. The liberal-communitarian debate. In: Taylor C (ed) Philosophical arguments. Harvard University Press, Cambridge MA London, S 20-34

35. Werner M (2003) Diskursethik als Maximenethik. Von der Prinzipienbegründung zur Handlungsorientierung. Königshausen \& Neumann, Würzburg

36. Werner M (2006) Diskursethik. In: Düwell M, Hübenthal C, Werner M (Hrsg) Handbuch Ethik. Metzler, Stuttgart Weimar, S 140-151 